\title{
Nonexistence of two-dimensional sessile drops in the diffuse-interface model
}

\author{
E. S. Benilov* \\ (Dated: September 27, 2021)
}

\begin{abstract}
The diffuse-interface model (DIM) is a widely used tool for modeling fluid phenomena involving interfaces - such as, for example, sessile drops (liquid drops on a solid substrate, surrounded by saturated vapor) and liquid ridges (two-dimensional sessile drops). In this work, it is proved that, surprisingly, the DIM does not admit solutions describing static liquid ridges. If, however, the vapor-to-liquid density ratio is small - as, for example, for water at room temperature - the ridges can still be observed as quasi-static states, as their evolution is too slow to be distinguishable from evaporation. Interestingly, the nonexistence theorem cannot be extended to axisymmetric sessile drops and ridges near a vertical wall, which are not ruled out.
\end{abstract}

\section{INTRODUCTION}

The diffuse-interface model (DIM) was developed in Refs. [1 6] and used for modeling various interfacial phenomena (e.g., Refs. [7-16] and references therein). Unlike phenomenological models, the DIM is based on a physical assumption: that the van der Waals intermolecular force is described by a pair-wise potential whose spatial scale is much smaller than that of the flow.

In the present work, the DIM is applied to a static liquid-vapor interface located near a flat horizontal substrate. The fluid between the substrate and interface is in liquid phase, whereas the fluid above the interface is vapor. In three dimensions, such a configuration is usually referred to as a "sessile drop", and in two dimensions, a "liquid ridge" (hereinafter, just "ridge").

The main conclusion of the present work will come as a surprise: the DIM does not admit solutions describing static ridges. The proof of the nonexistence theorem will be presented for the van der Waals fluid (Theorem 1), but it can be readily extended to any equation of state (Theorem 2). This result seems to contradict the existence of ridge solutions in the Navier-Stokes equations, but the apparent conflict will be convincingly resolved.

\section{FORMULATION}

Let a fluid's density field be two-dimensional and described by a function $\rho(x, z)$ where $x$ and $z$ are the horizontal and vertical coordinates, respectively. The following nondimensional variables will be used:

$$
\rho_{n d}=b \rho, \quad x_{n d}=\left(\frac{a}{K}\right)^{1 / 2} x, \quad z_{n d}=\left(\frac{a}{K}\right)^{1 / 2} z,
$$

where $a$ and $b$ are the van der Waals constants $\left(b^{-1}\right.$ is the maximum density), and $K$ is the Korteweg parameter

\footnotetext{
* Department of Mathematics and Statistics, University of Limerick, Limerick, V94 T9PX, Ireland; Eugene.Benilov@ul.ie https://staff.ul.ie/eugenebenilov/
}

(characterizing the fluid-fluid intermolecular force). A nondimensional temperature can be defined by

$$
\tau=\frac{R T b}{a},
$$

where $R$ is the gas constant and $T$, the dimensional temperature.

In the equilibrium case (zero flow, constant temperature), the DIM reduces to a single equation for the nondimensional density field (e.g., Eq. (34) of Ref. [15]). For the van der Waals fluid, this equation is (the subscript nd omitted)

$$
\tau\left(\ln \frac{\rho}{1-\rho}+\frac{1}{1-\rho}\right)-2 \rho-\frac{\partial^{2} \rho}{\partial x^{2}}-\frac{\partial^{2} \rho}{\partial z^{2}}=\mu,
$$

where $\mu$ is an undetermined constant. Note that, physically, the first two terms on the left-hand side of Eq. (1) represent the chemical potential of the van der Waals fluid.

Let the fluid be bounded below by a solid substrate located at $z=0$, in which case the DIM implies the following boundary condition [4, 15]:

$$
\rho=\rho_{0} \quad \text { at } \quad z=0,
$$

where $\rho_{0}$ characterizes the solid-fluid intermolecular force. Far above the substrate, $\rho$ tends to the density $\rho_{v}$ of saturated vapor,

$$
\rho \rightarrow \rho_{v} \quad \text { as } \quad z \rightarrow \infty .
$$

The vapor density $\rho_{v}$ and the matching liquid density $\rho_{l}$ are determined by the so-called Maxwell construction, comprising the requirements that the vapor's pressure and chemical potential be equal to those of the liquid (e.g., Ref. [15]). For the van der Waals fluid, the Maxwell construction is

$$
\begin{gathered}
\frac{\tau \rho_{v}}{1-\rho_{v}}-\rho_{v}^{2}=\frac{\tau \rho_{l}}{1-\rho_{l}}-\rho_{l}^{2}, \\
\tau\left(\ln \frac{\rho_{v}}{1-\rho_{v}}+\frac{1}{1-\rho_{v}}\right)-2 \rho_{v} \\
=\tau\left(\ln \frac{\rho_{l}}{1-\rho_{l}}+\frac{1}{1-\rho_{l}}\right)-2 \rho_{l} .
\end{gathered}
$$




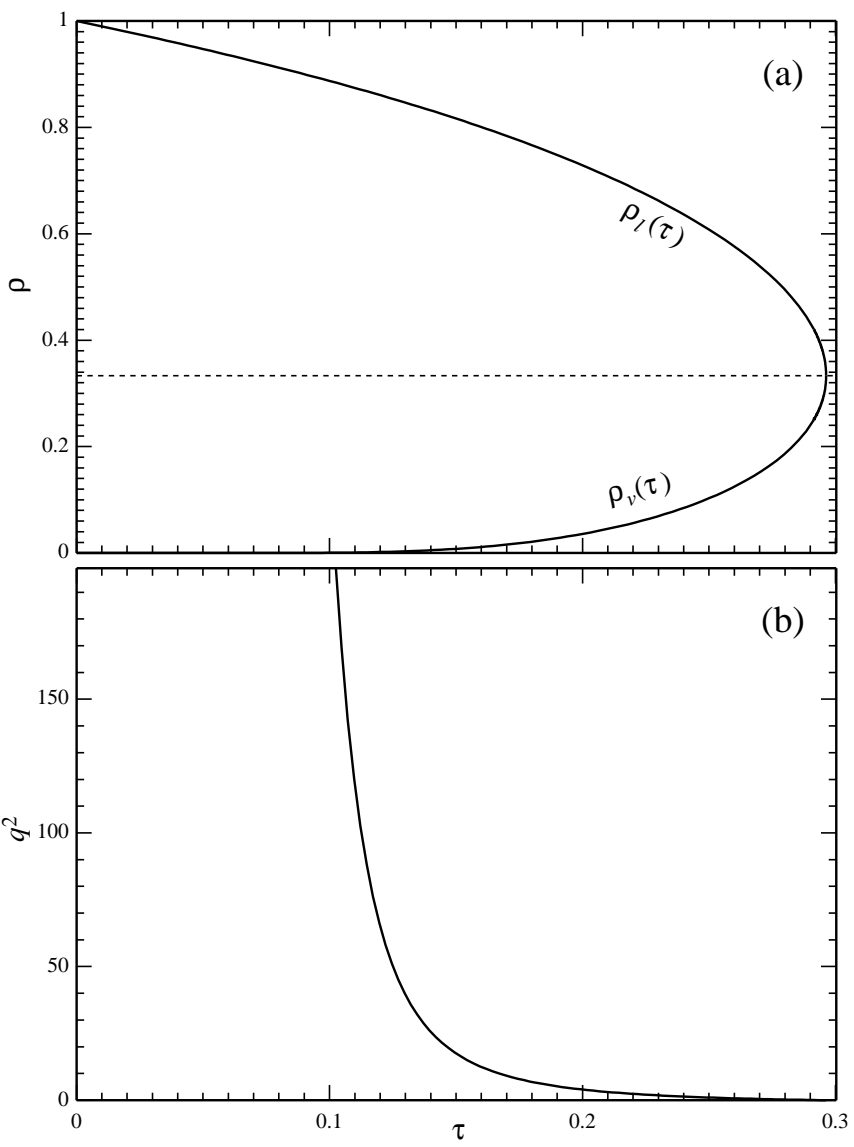

FIG. 1. The solution of the Maxwell construction (4)-(5) for the van der Waals fluid vs. the nondimensional temperature: (a) the densities of saturated vapor and liquid (the dotted line shows the critical density), (b) $q^{2}$ given by expression 14 .

Unlike $\rho_{v}$, the liquid density $\rho_{l}$ is not involved in the boundary-value problem for $\rho(x, z)$.

Eqs. (4)-(5) admit non-trivial $\left(\rho_{v} \neq \rho_{l}\right)$ solutions only if the temperature is lower than its critical value. Nondimensionally, this restriction amounts to $\tau<8 / 27$ and is implied everywhere in this paper (otherwise interfaces simply do not exist). The graphs of $\rho_{v}$ and $\rho_{l}$ vs. $\tau$ are shown in Fig. 11.

In principle, the boundary condition $(3)$ can be replaced with

$$
\rho \rightarrow \rho_{\infty} \quad \text { as } \quad z \rightarrow \infty
$$

where $\rho_{\infty}$ is not necessarily equal to $\rho_{v}$. What happens in this case depends on whether $\rho_{\infty}$ exceeds $\rho_{v}$. If it does, the vapor above the ridge is over-saturated - hence, unstable with respect to spontaneous formation of drops. Even if static solutions exist in this case, they are meaningless physically.

If, on the other hand, the vapor is under-saturated $\left(\rho_{\infty}<\rho_{v}\right)$, ridges cannot exist due to evaporation. This conclusion is based on physics, but can be readily confirmed mathematically, as this case is similar to the one actually examined $\left(\rho_{\infty}=\rho_{v}\right)$.
According to the DIM, ridges are accompanied by a precursor film stretching to infinity [4], so that

$$
\rho \rightarrow \bar{\rho}(z) \quad \text { as } \quad x \rightarrow \infty,
$$

where $\bar{\rho}(z)$ describes the vertical structure of the precursor film and satisfies the one-dimensional reduction of the boundary-value problem (1)-(3),

$$
\begin{gathered}
\tau\left(\ln \frac{\bar{\rho}}{1-\bar{\rho}}+\frac{1}{1-\bar{\rho}}\right)-2 \bar{\rho}-\frac{\mathrm{d}^{2} \bar{\rho}}{\mathrm{d} z^{2}}-\mu=0, \\
\bar{\rho}=\rho_{0} \quad \text { at } \quad z=0, \\
\bar{\rho} \rightarrow \rho_{v} \quad \text { as } \quad z \rightarrow \infty .
\end{gathered}
$$

One can safely assume that, if exists, the ridge solution is symmetric - say, with respect to $x=0$ - hence,

$$
\frac{\partial \rho}{\partial x}=0 \quad \text { at } \quad x=0 .
$$

To eliminate the trivial solution (describing the precursor film without a ridge), require

$$
\rho \neq \bar{\rho} \quad \text { at } \quad x=0 .
$$

This condition is sufficient if it holds in any non-zerolength interval of $z$, no matter how small. Finally, let

$$
\left|\int_{-\infty}^{\infty}(\rho-\bar{\rho}) \mathrm{d} x\right|<\infty \quad \forall z>0 .
$$

This condition eliminates solutions describing infinite arrays of ridges (as opposed to a single, isolated one).

\section{PROPERTIES OF THE PRECURSOR FILM}

The following properties of the function $\bar{\rho}(z)$ will be needed.

(i) Taking the limit $z \rightarrow \infty$ in Eq. (1) and recalling the boundary condition (3), one obtains

$$
\mu=\tau\left(\ln \frac{\rho_{v}}{1-\rho_{v}}+\frac{1}{1-\rho_{v}}\right)-2 \rho_{v} .
$$

Now, multiply Eq. 7 by $\mathrm{d} \bar{\rho} / \mathrm{d} z$ and integrate it with respect to $z$, which yields a separable first-order equation. Using the boundary condition (9) to fix the constant of integration in this equation and taking into account (13), one can extend $\bar{\rho}(z)$ to $z<0$, then show that it is monotonic, and eventually use the Maxwell construction (4)(5) to prove that

$$
\bar{\rho}(z) \rightarrow \rho_{l} \quad \text { as } \quad z \rightarrow-\infty .
$$

The extended $\bar{\rho}(z)$ describes a liquid-vapor interface in an unbounded space. Given its monotonicity, the boundary condition (8) may hold only if $\rho_{v}<\rho_{0}<\rho_{l}$. This requirement is implied everywhere in this paper. 
(ii) Using the above-mentioned separable equation, one can show that the solution of the boundary-value problem (7)-9 for $\bar{\rho}(z)$ is unique.

(iii) Introduce

$$
q^{2}=\frac{\tau}{\rho_{v}\left(1-\rho_{v}\right)^{2}}-2 .
$$

It can be deduced from the Maxwell construction (4)-(5) that

$$
q^{2}>0 \quad \text { if } \quad \tau<\frac{8}{27}
$$

(see also Fig. 1b). Then, it follows from (7), (13), and (9) that

$$
\bar{\rho}=\rho_{v}+\mathcal{O}\left(\mathrm{e}^{-q z}\right) \quad \text { as } \quad z \rightarrow \infty,
$$

where it is implied that $q>0$.

\section{THE NONEXISTENCE THEOREM}

The main result of the present work can be formulated as follows.

Theorem 1: The boundary-value problem (1)-(12) does not admit smooth solutions for $\rho(x, z)$.

Theorem 1 follows from the four lemmas formulated below. Lemmas 1-2 prove that, if a solution exists, it should satisfy a certain identity, whereas Lemmas 3-4 prove that the right-hand side of this identity is strictly smaller than its left-hand side. The resulting contradiction can only be resolved if Eqs. (1)-(12) do not admit any solutions.

Lemma 1: All solutions of Eqs. 11)-(12) (if exist) satisfy

$$
F[\rho(0, z)]=F[\bar{\rho}(z)],
$$

where the functional $F[\varrho(z)]$ is given by

$$
\begin{aligned}
F[\varrho(z)]=\int_{0}^{\infty}\left[\tau \varrho \ln \frac{\varrho}{1-\varrho}\right. & -\varrho^{2} \\
& \left.+\frac{1}{2}\left(\frac{\mathrm{d} \varrho}{\mathrm{d} z}\right)^{2}-\mu \varrho+p\right] \mathrm{d} z
\end{aligned}
$$

and

$$
p=\frac{\tau \rho_{v}}{1-\rho_{v}}-\rho_{v}^{2} .
$$

Note that, physically, $p$ is the vapor pressure far above the ridge.

To prove Lemma 1, multiply Eq. (1) by $\partial \rho / \partial x$ and integrate it with respect to $z$ from 0 to $\infty$. Integrating the term involving $\partial^{2} \rho / \partial z^{2}$ by parts, and using the boundary conditions $(2)-(3)$, one obtains

$$
\int_{0}^{\infty} \frac{\partial f}{\partial x} \mathrm{~d} z=0
$$

where

$$
f=\tau \rho \ln \frac{\rho}{1-\rho}-\rho^{2}-\frac{1}{2}\left(\frac{\partial \rho}{\partial x}\right)^{2}+\frac{1}{2}\left(\frac{\partial \rho}{\partial z}\right)^{2}-\mu \rho .
$$

Using the boundary condition (3) and expression 13 for $\mu$, one can show that $f \rightarrow-p$ as $z \rightarrow \infty$, with $f+p$ vanishing exponentially quickly (as follows from Lemma 2 proved below). This enables one to rewrite (18) in the form

$$
\frac{\partial}{\partial x} \int_{0}^{\infty}(f+p) \mathrm{d} z=0 .
$$

To obtain identity (17), one should integrate the above equality with respect to $x$ from 0 to $\infty$, replace $f$ with expression (19), and use conditions (6) and (10).

Lemma 2: Solutions of Eqs. (1)-12) (if exist) are such that

$$
\begin{aligned}
& \rho(x, z) \sim \rho_{v} \\
&+\frac{C}{z^{1 / 2}} \exp \left(-q z-\frac{q x^{2}}{2 z}\right) \quad \text { as } \quad z \rightarrow \infty,
\end{aligned}
$$

where $q>0$ is given by (14) and $C$ is a constant.

Let $\rho=\bar{\rho}+\tilde{\rho}$. Under the assumption that $\rho$ exists and satisfies Eq. (1), the large- $z$ asymptotics of $\tilde{\rho}(x, z)$ should vanish as $z \rightarrow \infty$ and satisfy the linearized version of Eq. (1),

$$
\begin{gathered}
\tilde{\rho} \rightarrow 0 \quad \text { as } \quad z \rightarrow \infty, \\
q^{2} \tilde{\rho}-\frac{\partial^{2} \tilde{\rho}}{\partial x^{2}}-\frac{\partial^{2} \tilde{\rho}}{\partial z^{2}}=0 \quad \text { as } \quad z \rightarrow \infty .
\end{gathered}
$$

The general solution of Eq. 22 subject to condition 21 can be found via the Fourier transformation with respect to $x$, which yields

$$
\begin{aligned}
\tilde{\rho} & \sim \int_{-\infty}^{\infty} B(k) \\
& \times \exp \left(-z \sqrt{q^{2}+k^{2}}+\mathrm{i} x k\right) \mathrm{d} k \quad \text { as } \quad z \rightarrow \infty
\end{aligned}
$$

where $B(k)$ is an undetermined function. By virtue of (12), $B(k)$ is continuous for all $k$ including $k=0-$ on the basis of which the above integral can be simplified and eventually evaluated,

$$
\tilde{\rho} \sim B(0) \sqrt{\frac{2 \pi q}{z}} \exp \left(-z q-\frac{q x^{2}}{2 z}\right) \quad \text { as } \quad z \rightarrow \infty .
$$

Recalling the definition of $\tilde{\rho}$ and letting $B(0) \sqrt{2 \pi q}=$ $C$, one can transform the above expression into 20 , as required.

Lemma 3: The function $\bar{\rho}(z)$ minimizes the functional $F[\varrho(z)]$ under additional constraints

$$
\varrho=\rho_{0} \quad \text { at } \quad z=0,
$$




$$
\varrho \rightarrow \rho_{v} \quad \text { as } \quad z \rightarrow \infty .
$$

To understand the role of Lemma 3 in proving Theorem 1 , note that the former and condition (11) make it impossible for identity (17) to hold - thus creating the desired contradiction.

Let $\delta \varrho(z)$ be a variation of $\varrho(z)$, so that the boundary conditions (23)-24 yield

$$
\begin{aligned}
& \delta \varrho=0 \quad \text { at } \quad z=0, \\
& \delta \varrho \rightarrow 0 \quad \text { as } \quad z \rightarrow \infty \text {. }
\end{aligned}
$$

Under these conditions, the requirement

$$
\delta F[\varrho(z)]=0
$$

yields an equation for $\varrho$, coinciding with Eq. (7) for $\bar{\rho}$. Conditions $(23)-(24)$, in turn, coincide with the conditions (8)-9 for $\bar{\rho}$. Thus, $\varrho=\bar{\rho}$ is a stationary point of $F[\varrho(z)]$.

Recall also that $\bar{\rho}(z)$ is unique - hence, $F[\varrho(z)]$ has only one stationary point. If it happens to be a minimum, it is the absolute minimum of the functional in question.

To ensure that $\varrho=\bar{\rho}$ is indeed a minimum of $F[\varrho(z)]$, one has to prove that

$$
\delta^{2} F[\varrho(z)]>0 \quad \text { for } \quad \varrho=\bar{\rho} .
$$

To do so, observe that

$$
\delta^{2} F[\varrho(z)]=\int_{0}^{\infty} \delta \varrho \hat{A} \delta \varrho \mathrm{d} z
$$

where

$$
\hat{A}=\frac{\tau}{\bar{\rho}(1-\bar{\rho})^{2}}-2-\frac{\mathrm{d}^{2}}{\mathrm{~d} z^{2}}
$$

is a second-order self-adjoint non-singular operator hence, its spectrum is real and the eigenfunctions form an orthogonal basis in $L^{2}(0, \infty)$ [17. Then, condition 25) holds if and only if $\hat{A}$ is positive-definite - which it indeed is, as follows from Lemma 4.

Lemma 4: All eigenvalues of $\hat{A}$ are strictly positive.

Let $\lambda$ be a continuous-spectrum eigenvalue of $\hat{A}$, and $\psi$ the corresponding eigenfunction,

$$
\begin{gathered}
{\left[\frac{\tau}{\bar{\rho}(1-\bar{\rho})^{2}}-2\right] \psi-\frac{\mathrm{d}^{2} \psi}{\mathrm{d} z^{2}}=\lambda \psi,} \\
\psi=0 \quad \text { at } \quad z=0, \\
\psi \sim \cos \left(\kappa_{c} z+\theta_{c}\right) \quad \text { as } \quad z \rightarrow \infty,
\end{gathered}
$$

where $\theta_{c}$ is an undetermined constant, $\kappa_{c}=\sqrt{\lambda-q^{2}}$ with $q^{2}$ given by 14 , and it is implied that $\kappa_{c}$ is real hence,

$$
\lambda \geq q^{2}
$$

Given 15, the above inequality entails $\lambda>0$ as required.

To examine the discrete spectrum (if it exists), assume that $\lambda<q^{2}$ and replace 28 with

$$
\psi=\mathcal{O}\left(\mathrm{e}^{-\kappa_{d} z}\right) \quad \text { as } \quad z \rightarrow \infty,
$$

where $\kappa_{d}=\sqrt{q^{2}-\lambda}$. Next, introduce $\phi(z)$ such that

$$
\psi=\frac{\mathrm{d} \bar{\rho}}{\mathrm{d} z} \phi
$$

Substituting this expression into $(26)-27$ and $(29)$, and recalling that $\bar{\rho}$ satisfies (7) and (16), one obtains

$$
\begin{gathered}
-\frac{\mathrm{d}}{\mathrm{d} z}\left[\left(\frac{\mathrm{d} \bar{\rho}}{\mathrm{d} z}\right)^{2} \frac{\mathrm{d} \phi}{\mathrm{d} z}\right]=\lambda\left(\frac{\mathrm{d} \bar{\rho}}{\mathrm{d} z}\right)^{2} \phi, \\
\phi=0 \quad \text { at } \quad z=0, \\
\phi=\mathcal{O}\left(\mathrm{e}^{-\kappa_{d} z+q z}\right) \quad \text { as } \quad z \rightarrow \infty
\end{gathered}
$$

Now, multiply Eq. (31) by $\phi$ and integrate it with respect to $z$ from 0 to $\infty$. Integrating by parts and taking into account conditions (32)- (33) and (16), one obtains

$$
\int_{0}^{\infty}\left(\frac{\mathrm{d} \varrho}{\mathrm{d} z}\right)^{2}\left(\frac{\mathrm{d} \phi}{\mathrm{d} z}\right)^{2} \mathrm{~d} z=\lambda \int_{0}^{\infty}\left(\frac{\mathrm{d} \varrho}{\mathrm{d} z}\right)^{2} \phi^{2} \mathrm{~d} z .
$$

Both integrals in the above identity are strictly positive and converge (the latter, because their integrands decay exponentially as $z \rightarrow \infty)$. Hence, $\lambda>0$, as required.

The proof of Theorem 1 is now complete. Next, it will be extended to an arbitrary equation of state (not necessarily the van der Waals one).

Theorem 2: Let smooth functions $G(\rho, \tau)$ and $p(\rho, \tau)$ be such that $\partial p / \partial \rho=\rho \partial G / \partial \rho$, and consider the following extension of Eq. (1):

$$
G(\tau, \rho)-\frac{\partial^{2} \rho}{\partial x^{2}}-\frac{\partial^{2} \rho}{\partial z^{2}}=\mu
$$

that of the Maxwell construction (4)-(5):

$$
G\left(\rho_{v}, \tau\right)=G\left(\rho_{l}, \tau\right), \quad p\left(\rho_{v}, \tau\right)=p\left(\rho_{l}, \tau\right),
$$

and that of the precursor film problem (7)-(9):

$$
\begin{gathered}
G(\tau, \bar{\rho})-\frac{\mathrm{d}^{2} \bar{\rho}}{\mathrm{d} z^{2}}-\mu=0, \\
\bar{\rho}=\rho_{0} \quad \text { at } \quad z=0, \\
\bar{\rho} \rightarrow \rho_{v} \quad \text { as } \quad z \rightarrow \infty .
\end{gathered}
$$

(34)-(38) and the (old) boundary conditions (2)-(3), (6), (10)-(12), do not admit smooth solutions describing liquid ridges. 
The proof of Theorem 2 will not be presented, as it is similar to that of Theorem 1, e.g., the van der Waals expression (14) should be replaced with

$$
q^{2}=\left(\frac{\partial G}{\partial \rho}\right)_{\rho=\rho_{v}}
$$

etc. Thus, the DIM does not admit solutions for an isolated static ridge in any fluid.

\section{DISCUSSION}

The most counter-intuitive aspect of Theorems 1-2 is that it rules out a phenomenon which one can easily reproduce by depositing a streak of water on one's kitchen table. There seems to be a mathematical paradox too: ridges do exist in the Navier-Stokes equations, which can be viewed as a liquid-only, incompressible reduction of the DIM.

The resolution of the paradox is best illustrated under the assumption that the interfacial slope $\varepsilon$ is small, i.e., in the thin-film approximation. The study of this limit [18] shows that the ridge dynamics depends on how the vapor-to-liquid density ratio $\rho_{v} / \rho_{l}$ compares to $\varepsilon$ :

- If $\rho_{v} / \rho_{l} \gtrsim \varepsilon^{4 / 3}$, one can derive a thin-film approximation of the DIM and identify the terms in it that disallow the ridge solutions.

- If $\rho_{v} / \rho_{l} \ll \varepsilon^{4 / 3}$, these terms vanish, and the resulting thin-film version of the DIM coincides with that of the Navier-Stokes equations (both admit ridge solutions).
Note that, for common fluids at room temperature, $\rho_{v} / \rho_{l}$ is indeed very small: for water at $T=20^{\circ} \mathrm{C}$, for example, $\rho_{v} / \rho_{l} \approx 1.7 \times 10^{-5}$. Thus, one can conjecture that the observed ridges are quasi-static: they do evolve, but their evolution is too slow to be distinguishable from evaporation. It still remains to find out how exactly they evolve, which seems impossible to predict using qualitative arguments.

Besides, water on a table is surrounded by air, not by water vapor as in the present formulation. It is not obvious that Theorem 1 can be generalized for a mixture of fluids - especially, if the temperature happens to be supercritical for some of the components (the critical point of nitrogen is $-147^{\circ} \mathrm{C}$, and that of oxygen is $-119^{\circ} \mathrm{C}$ ).

Interestingly, Theorems 1-2 cannot be extended (at least, not in a simple way) to axisymmetric sessile drops, described by $\rho(r, z)$, where $r$ is the polar radius. To understand why, consider the axisymmetric equivalent of the (two-dimensional) identity (17),

$$
F[\rho(0, z)]-\int_{0}^{\infty} \int_{0}^{\infty} \frac{1}{r}\left(\frac{\partial \rho}{\partial r}\right)^{2} \mathrm{~d} z \mathrm{~d} r=F[\bar{\rho}(z)]
$$

Comparing (34) to (17), one can see that the extra term in the former eliminates the contradiction with the fact that the right-hand side of (39) is always smaller than the first term on its left-hand side.

There is another setting for which Theorems 1-2 cannot be easily generalized: if a vertical wall is present.
[1] P. C. Hohenberg and B. I. Halperin, Theory of dynamic critical phenomena, Rev. Mod. Phys. 49, 435 (1977).

[2] H. Gouin, Utilization of the Second Gradient Theory in continuum mechanics to study the motion and thermodynamics of liquid-vapor interfaces, in Physicochemical Hydrodynamics, NATO ASI Series, Vol. 174, edited by M. G. Velarde (Springer US, 1987) pp. 667-682.

[3] D. M. Anderson, G. B. McFadden, and A. A. Wheeler, Diffuse-interface methods in fluid mechanics, Annu. Rev. Fluid Mech. 30, 139 (1998).

[4] L. M. Pismen and Y. Pomeau, Disjoining potential and spreading of thin liquid layers in the diffuse-interface model coupled to hydrodynamics, Phys. Rev. E 62, 2480 (2000)

[5] D. Jacqmin, Contact-line dynamics of a diffuse fluid interface, J. Fluid Mech. 402, 57 (2000)

[6] U. Thiele, S. Madruga, and L. Frastia, Decomposition driven interface evolution for layers of binary mixtures. I. Model derivation and stratified base states, Phys. Fluids 19, 122106 (2007).

[7] H. Ding and P. D. M. Spelt, Wetting condition in diffuse interface simulations of contact line motion, Phys. Rev.
E 75, 046708 (2007)

[8] P. Yue and J. J. Feng, Can diffuse-interface models quantitatively describe moving contact lines?, Eur. Phys. J. Spec. Top. 197, 37 (2011).

[9] D. N. Sibley, A. Nold, N. Savva, and S. Kalliadasis, The contact line behaviour of solid-liquid-gas diffuse-interface models, Phys. Fluids 25, 092111 (2013).

[10] H. Kusumaatmaja, E. J. Hemingway, and S. M. Fielding, Moving contact line dynamics: from diuse to sharp interfaces, J. Fluid Mech. 788, 209 (2016).

[11] A. Fakhari and D. Bolster, Diffuse interface modeling of three-phase contact line dynamics on curved boundaries: A lattice Boltzmann model for large density and viscosity ratios, J. Comput. Phys. 334, 620 (2017).

[12] R. Borcia, I. D. Borcia, M. Bestehorn, O. Varlamova, K. Hoefner, and J. Reif, Drop behavior influenced by the correlation length on noisy surfaces, Langmuir 35, 928 (2019)

[13] M. Gallo, F. Magaletti, D. Cocco, and C. M. Casciola, Nucleation and growth dynamics of vapour bubbles, J. Fluid Mech. 883, A14 (2020).

[14] E. J. Gelissen, C. W. M. van der Geld, M. W. Baltussen, 
and J. G. M. Kuerten, Modeling of droplet impact on a heated solid surface with a diffuse interface model, Int. J. Multiphase Flow 123, 103173 (2020).

[15] E. S. Benilov, The dependence of the surface tension and contact angle on the temperature, as described by the diffuse-interface model, Phys. Rev. E 10.1103/PhysRevE.101.042803 (2020).

[16] E. S. Benilov, Asymptotic reductions of the diffuse- interface model, with applications to contact lines in fluids, to appear in Phys. Rev. Fluids (also available on https://arxiv.org/abs/1907.04434) (2020).

[17] R. Courant and D. Hilbert, Methods of Mathematical Physics (Wiley, 1989).

[18] E. S. Benilov, Dynamics of liquid films, as described by the diffuse-interface model, https://arxiv.org/abs/2007. 03351 (2020). 